\title{
Synovial White Cell Count in the Diagnosis of Septic Arthritis: Are Current Diagnostic Practices Appropriate?
}

\author{
Kalpa Perera \\ Department of Surgery, Eastern Health, Box Hill, Australia \\ Email: kalpagperera@gmail.com
}

Received 5 November 2015; accepted 19 December 2015; published 22 December 2015

Copyright (C) 2015 by author and Scientific Research Publishing Inc. This work is licensed under the Creative Commons Attribution International License (CC BY). http://creativecommons.org/licenses/by/4.0/ (c) (7) Open Access

\section{Abstract}

Introduction \& aims: Septic arthritis is an emergency, potentially causing irreversible joint destruction and disability. Synovial WCC and polymorphonuclear cell percentage are the best predictors of septic arthritis likelihood. Yet, synovial white cell and differential count are not routinely assessed. We aim to investigate the incidence of failure to perform these tests, and to develop correct synovial fluid analysis practices. Method: This is a retrospective analysis of native joints having undergone arthrocentesis for suspicion of septic arthritis at Box Hill Hospital (BHH) during September 2011 and September 2013 inclusive. Recruitment was from the Eastern Health Decision Support Service (DSS), a database compiled from all systems within Eastern Health, of which BHH is a member. The study was limited to large joints, including hip, knee and shoulder. All prosthetic joints were excluded from the patient population. All patient histories were examined for suspicion of septic arthritis and subsequent arthrocentesis. Pathology records were accessed to determine incidence of cell count and differential. Results: One hundred and thirty-six cases of joint aspirations were identified within the time frame, of which sixty-seven fitted our criteria for evaluation. All but two cases were delivered using the DSS, which was limited to data compiled only until June 2013. The two remaining cases were identified with a manual search of the radiology and pathology databases from June to September 2013. 22 of the 67 joint aspirates studied did not have a cell count carried out. Four of these 22 cases had a diagnosis of septic arthritis. In five aspirates, there was a failure to confirm a definite diagnosis and they were thus conservatively treated as a septic joint. The remaining acute joints in which no cell count was done were gout ( 7 cases), pseudogout ( 5 cases) and rheumatoid arthritis (1 case). Cell counts were not routinely detected for a variety of reasons. Eleven aspirates were deemed too viscous, and in eight cases the sample had clotted prior to pathologist assessment. Two cases had insufficient volume, and one sample was too bloodstained to calculate a cell count and differential; likely due to traumatic aspiration. Conclusions: $33 \%$ of acute monoarthritis' evaluated over the study period failed to have a synovial fluid WCC and differential. This may be due to inadequate samples, or lack of 
appropriate collection tube. Better education is required for appropriate collection and test requesting wherein a diagnosis of septic arthritis is in question.

\title{
Keywords
}

\author{
Septic Arthritis, Arthrocentesis, Synovial White Cell Count
}

\section{Introduction}

Septic arthritis is a medical emergency that can deteriorate rapidly, leading to irreversible joint destruction and disability [1]. The incidence of septic arthritis has been reported at 5.7 to 9 per 100,000 person-years in developed nations [2] [3]. Prompt diagnosis and management are vital in preventing morbidity and $11 \%$ mortality [4]. The key to diagnosis is a high clinical suspicion with the primary adjunct of synovial fluid analysis. Blood culture and serum investigations are also useful, particularly in monitoring response to treatment. In addition, plain films are commonly taken, although they are mostly non-specific at the diagnostic stage [5]. Current practice is to assess synovial fluid for white cell count (WCC) with differential, Gram stain, and culture [6]. Chiefly, synovial WCC and percentage of polymorphonuclear are the best predictors of likelihood of septic arthritis whilst awaiting pending culture [7]. Nonetheless, it has been our observation that synovial fluid analysed with the suspicion of septic arthritis has not been routinely assessed for WCC and differential. Whether this is failure of clinician documentation or the pathologist in completing the analysis is unknown. In our study, we aim to investigate the incidence of this in our institution and subsequently develop a framework for what the clinician should request for synovial fluid analysis.

\section{Methods}

A retrospective study was performed on native joints having undergone arthrocentesis with the suspected diagnosis of septic arthritis at Box Hill Hospital between September 2011 and September 2013 inclusive. This timeframe was selected, as it is the extent to which the scanned medical records extend historically. The major source of patient recruitment was the Eastern Health Decision Support Service (DSS), who maintains a central data warehouse compiled from all hospital systems within Eastern Health; the health network that Box Hill Hospital is a part of. The Eastern Health Office of Research and Ethics granted appropriate approval to conduct this study.

The study was limited to large joints; this includes hip, knee and shoulder. All prosthetic joints were excluded from the patient population.

All patient histories were examined for suspicion of septic arthritis and completion of arthrocentesis. Of those with confirmed joint aspiration, pathology records were accessed to determine incidence of cell count and differential.

\section{Results}

One hundred and thirty-six cases of joint aspirations were identified within the time frame, of which sixty-seven fit our criteria for evaluation. All but two cases were delivered using the DSS, which was limited to data compiled only until June 2013. The two remaining cases were identified with a manual search of the radiology and pathology databases from June to September 2013.

22 of the 67 joint aspirates studied did not have a cell count carried out. Four of these 22 cases had a diagnosis of septic arthritis. In five aspirates, there was a failure to confirm a definite diagnosis and were thus conservatively treated as a septic joint. The remaining acute joints in which no cell count was done were gout (7 cases), pseudogout (5 cases) and rheumatoid arthritis (1 case).

Cell counts were not routinely detected for a variety of reasons. Eleven aspirates were deemed too viscous, and in eight cases the sample had clotted prior to pathologist assessment. Two cases had insufficient volume of less than $2 \mathrm{ml}$, and one sample was too bloodstained to calculate a cell count and differential; likely due to traumatic aspiration. 


\section{Discussion}

Evaluation of an acutely painful, swollen joint requires a multifaceted approach utilising a patient's risk factors, history and presenting physical signs in addition to adjunctive laboratory tests to determine an accurate diagnosis and appropriate management.

Whilst awaiting definitive synovial fluid culture and gram stain results, the synovial WCC and percentage of polymorphonuclear cells from joint aspiration provide the best predictor of septic arthritis [7].

22 (33\%) of 68 acute monoarthritis presenting to our emergency department over the study period failed to have a synovial fluid WCC and differential. Yet, $41 \%$ of these cases were subsequently managed as septic arthritis. High viscosity, bloodstained, and clotted specimens constituted $91 \%$ of cases without cell count performed. This may be due to inadequate samples taken, or lack of appropriate collection tube. Clotted specimens suggest poor communication between the clinician and the laboratory. Clinicians must be advised to communicate in a timely manner with laboratory staff to expedite analysis of the sample.

Nonetheless, controversy remains regarding use of synovial cell counts, especially in determining appropriate cut-off values. In accordance with established guidelines, our institution uses 50,000/mm 3 as a minimum synovial WCC for diagnosis of septic arthritis. Yet, the literature contains no true consensus regarding this figure. A retrospective study in 2002 looked at 202 patients with suspected septic arthritis [8]. Those with a synovial fluid WCC of more than 50,000/mm 3 had a proven diagnosis of sepsis in $47 \%$ of cases. Those with a synovial fluid WCC of more than 100,000/mm 3 had the diagnosis confirmed in $77 \%$ of cases. The authors concluded that, although a synovial fluid WCC of less than 50,000/mm 3 reduced the likelihood of the diagnosis of sepsis, it could not rule it out conclusively. Further, a systematic review suggested significant heterogeneity in sensitivity and specificity with synovial fluid analysis [9].

Although not a feature in our study group, the lack of a cell count may not only delay treatment but also result in unnecessary operative procedures. In a critical appraisal of the literature, Margaretten et al. found a consistently reproducible diagnostic accuracy of synovial fluid WCC [7]. Arthrocentesis, being a relatively simple procedure with minimal risk [10], thus has great bearing on the immediate management of the patient.

The main limitation of our study is the small sample size, potentially limiting generalizability. We essentially performed an analysis of a snapshot of one institution. Practices across different centres may vary widely depending on a number of factors, including logistics of specimen collection and delivery, and training of clinician.

\section{Conclusion}

Synovial WCC and cell differential are effective and relatively simple tests for identifying septic arthritis which awaits results of Gram stain and culture. Unfortunately, the high proportion (33\%) of cases in our study that did not undergo this test would suggest that it was not being properly utilised. Better education is required for appropriate collection and test requesting wherein a diagnosis of septic arthritis is in question.

\section{References}

[1] Weston, V.C., Jonesa, A.C., Bradbury, N., et al. (1999) Clinical Features and Outcome of Septic Arthritis in a Single UK Health District 1982-1991. Annals of the Rheumatic Diseases, 58, 214-219. http://dx.doi.org/10.1136/ard.58.4.214

[2] Kaandorp, C.J., et al. (1997) Incidence and Sources of Native and Prosthetic Joint Infection: A Community Based Prospective Survey. Annals of the Rheumatic Diseases, 56, 470-475. http://dx.doi.org/10.1136/ard.56.8.470

[3] Morgan, D.S., Fishera, D., Merianos, A., et al. (1996) An 18-Year Clinical Review of Septic Arthritis from Tropical Australia. Epidemiology and Infection, 117, 423-428. http://dx.doi.org/10.1017/S0950268800059070

[4] Gupta, M.N., Sturrock, R.D. and Field, M. (2001) A Prospective 2-Year Study of 75 Patients with Adult-Onset Septic Arthritis. Rheumatology (Oxford), 40, 24-30. http://dx.doi.org/10.1093/rheumatology/40.1.24

[5] Rousseau, I., Cardinal, É., Raymond-Tremblay, D., et al. (2001) Gout: Radiographic Findings Mimicking Infection. Skeletal Radiology, 30, 565-569. http://dx.doi.org/10.1007/s002560100392

[6] Wheeless, C.R. (2012) Septic Arthritis: General Orders and Treatment. Wheeless' Textbook of Orthopaedics 2012, Data Trace Internet Publishing, North Carolina, USA.

[7] Margaretten, M.E., Kohlwes, J., Moore, D., et al. (2007) Does This Adult Patient Have Septic Arthritis? JAMA: The Journal of the American Medical Association, 297, 1478-1488. http://dx.doi.org/10.1001/jama.297.13.1478 
[8] Coutlakis, P.J., Roberts, W.N. and Wise, C.M. (2002) Another Look at Synovial Fluid Leukocytosis and Infection. Journal of Clinical Rheumatology, 8, 67-71. http://dx.doi.org/10.1097/00124743-200204000-00001

[9] Carpenter, C.R., Schuur, J.D., Everett, W.W., et al. (2011) Evidence-Based Diagnostics: Adult Septic Arthritis. Academic Emergency Medicine, 18, 781-796. http://dx.doi.org/10.1111/j.1553-2712.2011.01121.x

[10] Geirsson, A.J., Statkevicius, S. and Vikingsson, A. (2008) Septic Arthritis in Iceland 1990-2002: Increasing Incidence Due to Iatrogenic Infections. Annals of the Rheumatic Diseases, 67, 638-643.

http://dx.doi.org/10.1136/ard.2007.077131 